\title{
Lessons Learned during Thermal Hardware Integration on the Global Precipitation Measurement Satellite
}

\author{
Christine E. Cottingham \\ Edge Space Systems, Glenelg, MD, 21737 \\ and \\ Vivek H. Dwivedi, Carlton Peters, Daniel Powers, and Kan Yang \\ Goddard Spaceflight Center, Greenbelt, MD, 20770
}

\begin{abstract}
The Global Precipitation Measurement mission is a joint NASA/JAXA mission scheduled for launch in early 2014. The integration of thermal hardware onto the satellite began in the Fall of 2010 and will continue through the Summer of 2012. The thermal hardware on the mission included several constant conductance heat pipes, heaters, thermostats, thermocouples, radiator coatings, and blankets. During integration several problems arose and insights were gained that would help future satellite integrations. Also lessons learned from previous missions were implemented with varying degrees of success. These insights can be arranged into three categories. 1) The specification of flight hardware using analysis results. 2) The integration of thermal flight hardware onto the spacecraft. 3) The preparation and implementation of testing the thermal flight hardware via touch tests, resistance measurements and thermal vacuum testing.
\end{abstract}

\section{Nomenclature}

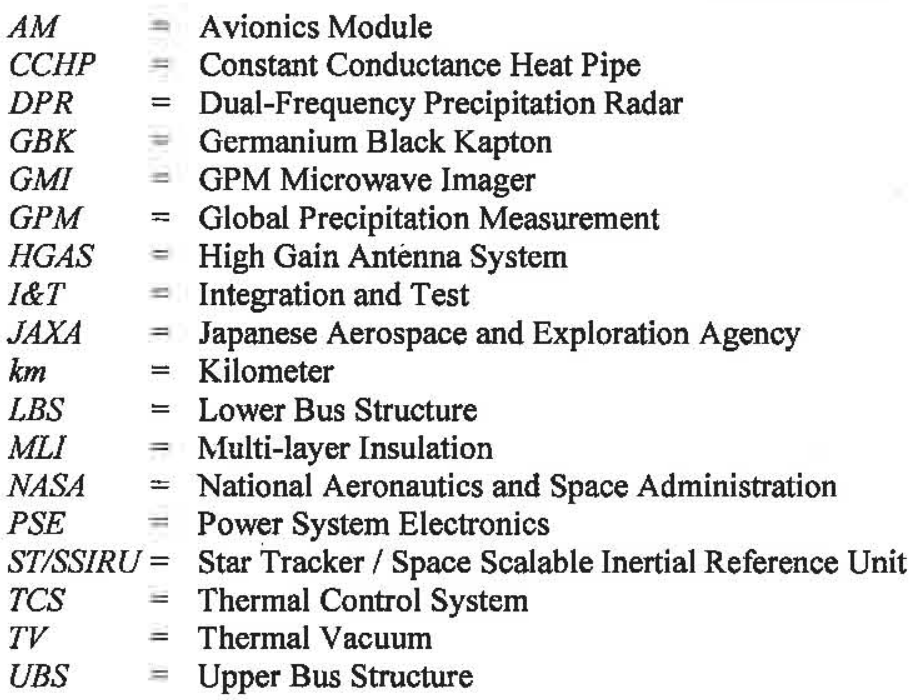

\section{Introduction}

The Global Precipitation Measurement (GPM) mission has a core satellite which is a joint NASA/JAXA 1 spacecraft scheduled for launch in early 2014. The GPM mission objectives are to advance the precipitation measurement capability from space which will improve climate modeling and prediction as well as our understanding of the water cycle. The mission will have a constellation of spacecraft to provide global precipitation measurement coverage. The core spacecraft's instruments are a microware radiometer (GMI) and a dual-frequency 
precipitation radar (DPR). The spacecraft will fly at a $65 \mathrm{deg}$ inclination, $400 \mathrm{~km}$ altitude orbit for a three year mission with a goal of a five year life.
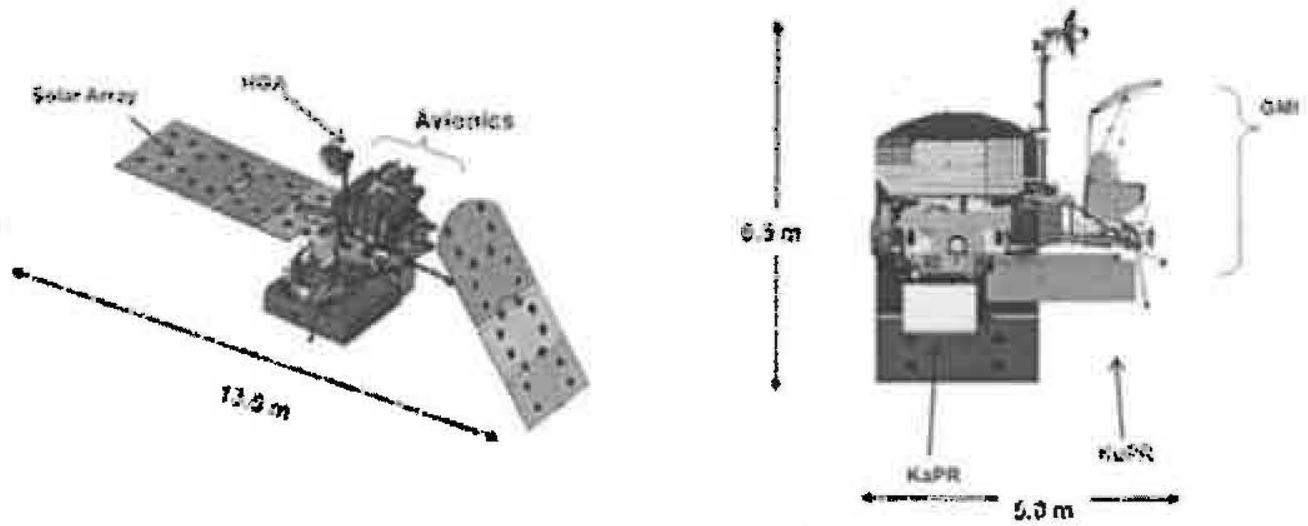

Figure 1. Overview of the GPM Observatory

\section{Brief Overview of the GPM Thermal Design}

The GPM Thermal Control Subsystem (TCS) is required to maintain all parts of the GPM Core Observatory within required temperature limits. This requirement is met by using a combination of thermal control hardware elements including dedicated high emittance radiators, constant conductance heat pipes (CCHPs), heat straps, heaters (software and mechanical), coatings, insulation, etc. to maintain equipment within limits. The TCS requires the Core Observatory to keep direct sunlight off the $+Y$ side of the spacecraft always during normal mission operations and as much as possible at all other times. This being so, all of the radiators are oriented $+Y$ with few exceptions. The major components on the GPM observatory as well as the axes of the spacecraft are shown in Figure 2. As seen from the figure, the ram directions are both $+X$ and $-X$, since the observatory executes a yaw flip at Beta $0^{\circ}$ to ensure that $+Y$ side is always anti-sun.

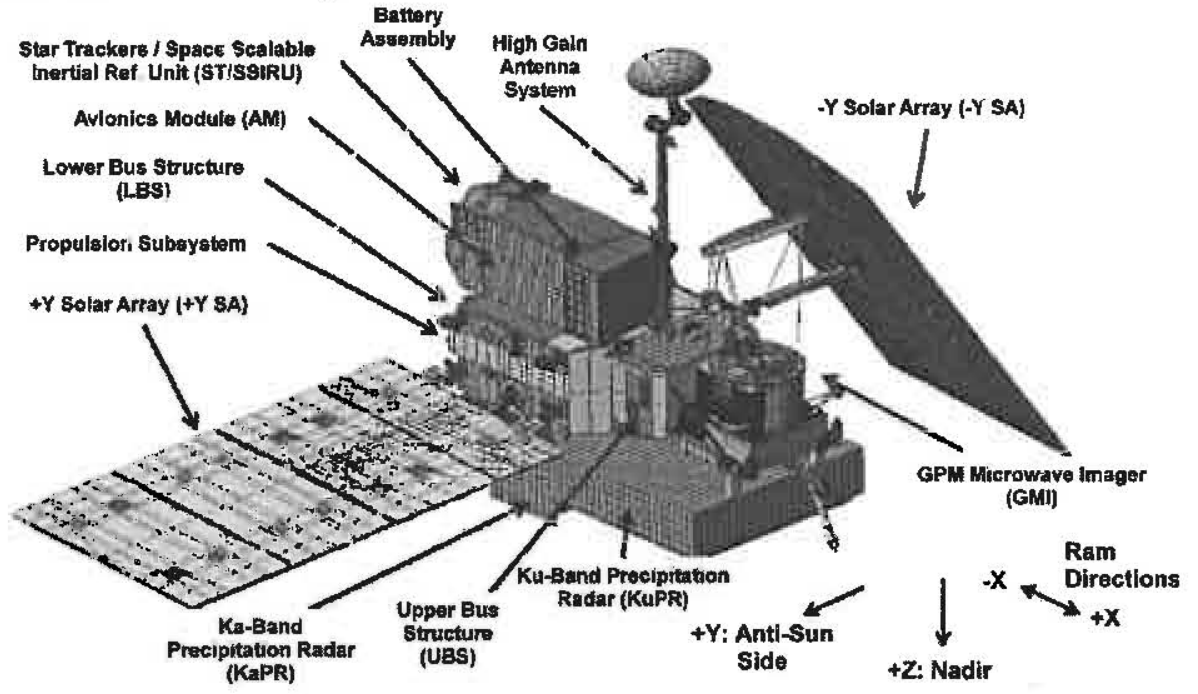

Figure 2. Major Components on the GPM Observatory

Most power dissipating components on GPM reject waste heat through their baseplates and so are generally mounted to structural surfaces using a thermal interface material to improve heat transfer. Examples include the avionics boxes in the Avionics Module (AM), the Reaction Wheel Assemblies (RWAs) in the Lower Bus Structure (LBS), and various constituent elements of the Star Tracker / Space Scalable Inertial Reference Unit (ST/SSIRU) 
assembly. NuSil CV 2946 gap filler and eGraf HITHERM 1210 are used for these components. Cho-Therm 1671 is used under the batteries. The AM and LBS structural surfaces transport the waste heat from the aforementioned power dissipating components via heat pipes: the AM has one U-shaped and one S-shaped embedded heat pipe, as well as two L-shaped heat pipes underneath the Power System Electronics (PSE) box, to transport heat to the avionics radiator; the batteries have four dedicated CCHPs to transport heat to the pocketed battery radiator. The LBS has two heat pipes to transport heat from the RWAs to the RWA Radiator; the Solar Array Drive Assemblies (SADAs) which are mounted to either Y-side of the LBS have their own respective radiators to reject heat. On the Upper Bus Structure (UBS), The RF components reject directly to a dedicated RF radiator, and the High-Gain Antenna System (HGAS) rejects heat through various radiating surfaces distributed along its assembly. The Major radiating surfaces on GPM are shown in Figure 3.

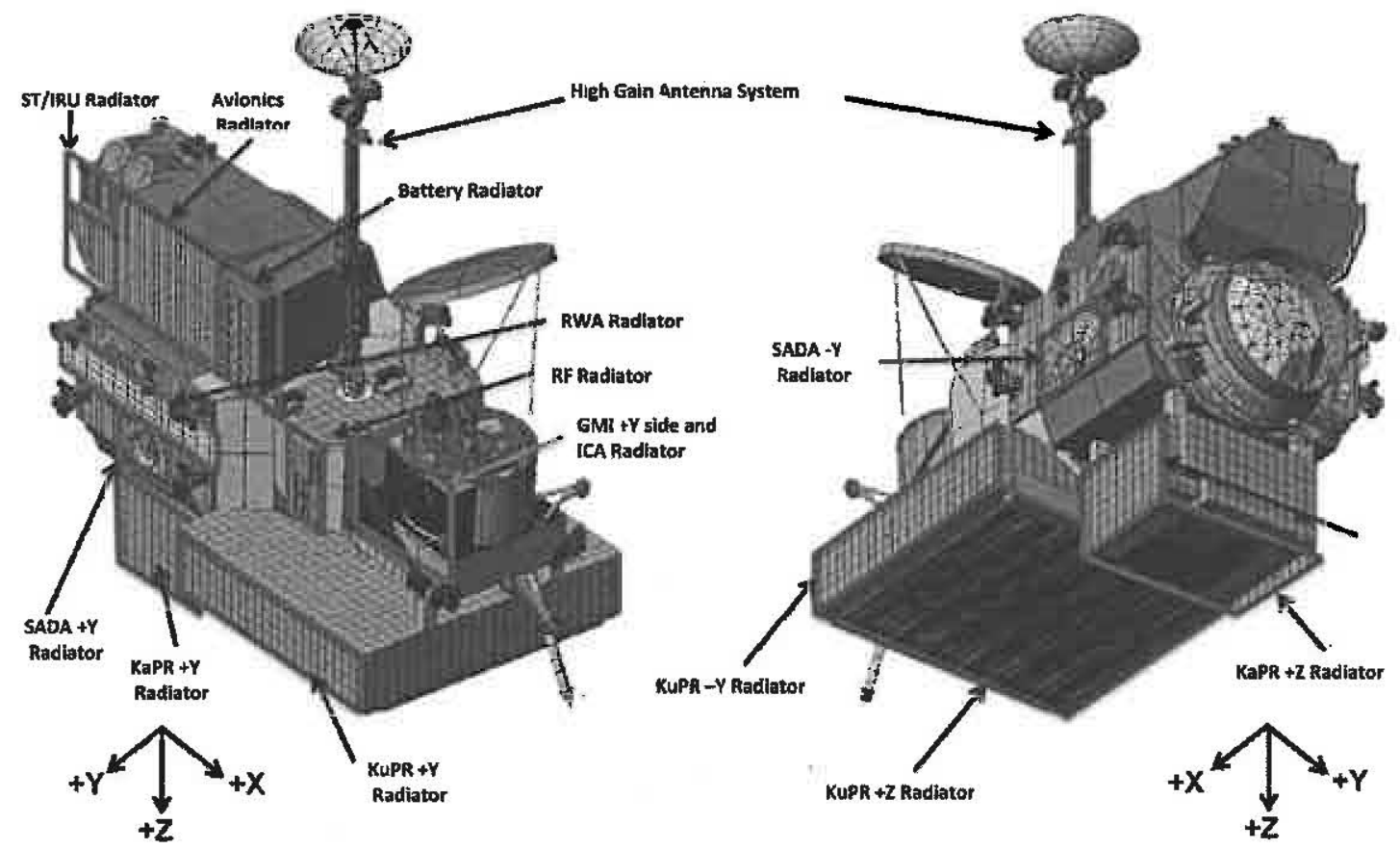

Figure 3. Major Radiating Surfaces on the GPM Observatory

Thermal multi-layer insulation (MLI) is used widely to minimize power loss to, or gain from, the environment. The MLI outer layer external to the spacecraft must thermally insulate and also resist surface electrical charging and atomic oxygen erosion. The MLI outer layer is Germanium Black Kapton (GBK) in order to achieve the necessary surface electrical conductivity and thermal performance. For the $-Z$ side of the $A M$, the $+Z$ side of the LBS, the areas enclosed by the UBS truss structure, and the skirt underneath the GMI instrument, blanket tents are employed largely to protect internal components from environmental influence. All other non-radiating surfaces mainly employ tight, form-fitting blankets for this purpose.

The TCS maintains science instrument mounting interfaces within required temperature limits: this includes the interface to the DPR, which consists of the Ka-band Preciptation Radar (KaPR) and the Ku-band Preciptation Radar $(\mathrm{KuPR})$; and the GPM Microwave Imager (GMI). The DPR instrument mounting interface requirements are met by using low thermal conductivity kinematic flexures so that even large variations in spacecraft flexure temperatures alter DPR critical components by no more than a few degrees. The GMI instrument mounting interface temperature requirement is a specific minimum temperature which is met by using thermostatically controlled heaters on the GMI flexures. However, thermal control internal to the science instruments are provided by the respective instrument vendors and are not handled via the Spacecraft Bus TCS. 


\section{GPM Thermal I\&T Lessons Learned}

Integration and Test (I\&T) of a spacecraft has several stages. 1) The specification of flight hardware using analysis results. 2) The integration of thermal flight hardware onto the spacecraft. 3) The preparation and implementation of testing the thermal flight hardware via touch tests, resistance measurements and thermal vacuum testing. Throughout this process, a range of issues were encountered that provide "lessons learned" for application on future spacecraft integrations.

\section{A. Lessons Learned During the Specification of Thermal Flight Hardware}

When ordering the constant conductance heatpipes for the spacecraft a detailed requirements document was generated. It detailed all of the thermal flight and test requirements. The shipping container for the pipes was a secondary thought. Since receiving the heatpipes we have run into a lot of difficulty finding room to store the flight spare heat pipes due to the room they take up as well has the ammonia being a hazardous gas. The shipping containers were made large, relatively flat, non-cleanroom compatible and unstackable. Lesson Learned: Specify shipping containers for heat pipes that are easier to store, such as a vertical box or a box with a minimal footprint.

Iridite is a common passivating coating used on aluminum parts on spacecraft. When bonding to an iridited surface, the primer must be removed. When ordering hardware such as heatpipes or when a metallic structure is being fabricated it is better to request a BR127 primer as the passivating coating. This primer is better than iridite because it has a wider temperature range as well as it does not need to be removed when items are bonded onto it. If electrical conductivity is needed, BR127C primer is conductive. The one disadvantage to using this coating is that it tends to be more expensive than iridite. It can be argued that the time saved during bonding hardware makes up for the increased cost. Most of the heat pipes on GPM were ordered with an iridite coating except for one pair of heatpipes that needed the wider temperature range that BR127 had. The bonding of temperature sensors and nutplates to the pipes was much easier and faster on the BR127 coated pipe. Lesson Learned: For metallic surfaces that will need to be bonded to, BR127 primer is more desirable as a passivating coating than Iridite.

When ordering heaters or thermostats, minimize the different part numbers ordered and used. For example, on GPM the thermostat setpoints for an actuator motor was $8^{\circ} \mathrm{C}$ to $13^{\circ} \mathrm{C}$ and thermostats in a different location needed to be $7^{\circ} \mathrm{C}$ to $14^{\circ} \mathrm{C}$. Since the typical resolution on the thermostat setpoint tends to be roughly $+/-2^{\circ} \mathrm{C}$ you could order the same part number for both locations and sort them by actual switching temperatures as provided by the vendor. Similar is true for heaters. Lesson Learned: If a conscious effort is made to use several of one type of heater or thermostat for multiple locations it simplifies the ordering processes as well as minimizes the number of flight spares that is needed.

\section{B. Lessons Learned during the integration of thermal flight hardware}

As a result of the thermal design of GPM, mechanical integration of the avionics radiator required that its reverse facesheet be simultaneously bolted to the flexures which provide structural support, as well as the heat pipe interfaces which transport heat from the avionics boxes to the radiator. Furthermore, since the reverse facesheet of the radiator is facing the avionics module cavity, there is no access to this face after it has been installed. Hence, during the integration of the avionics radiator to a mockup of the flight avionics module for a thermal risk-reduction test, it was necessary to have the mounting and wire-routing of all thermal hardware components on this facesheet completed before the mechanical integration. This included Kapton film heaters, thermistors, test thermocouples, and eGraf HITHERM-1210 sheets which provided the thermal interface between the radiator and the avionics heat pipes. During the mounting of the thermal hardware, full-size paper mockups of the "stay-out zone", i.e. the area in which the reverse facesheet of the radiator contacted the heat pipes, were taped onto the radiator to ensure that the bonded thermal hardware locations would not conflict with these interfaces. However, less consideration was made towards the direction in which the wires routed from the thermal hardware. During the mechanical integration, it was noticed that two thermistors had wires which routed too close to the heat pipe interface, and therefore were 
crushed by the L-bend of the heat pipes, as shown in Figure X. Moreover, one thermocouple had its epoxy bead too close to the heat pipe interface, thus causing an obstruction and preventing the heat pipe to be mounted flush with the radiator facesheet. Hence, the interfering thermistors had to be removed and the epoxy scraped off before new thermistors could be bonded, with care taken to route the wires in another direction that did not interfere with the heat pipe interface. As a result, there was a delay to the radiator integration and thermal risk-reduction test schedule. Lesson Learned: When mounting thermal hardware in areas with space restrictions or "stay-out zones", care should be taken to specify the direction in which the wires route from the thermal hardware such that the risk of interference with other future hardware to be mounted is minimized.

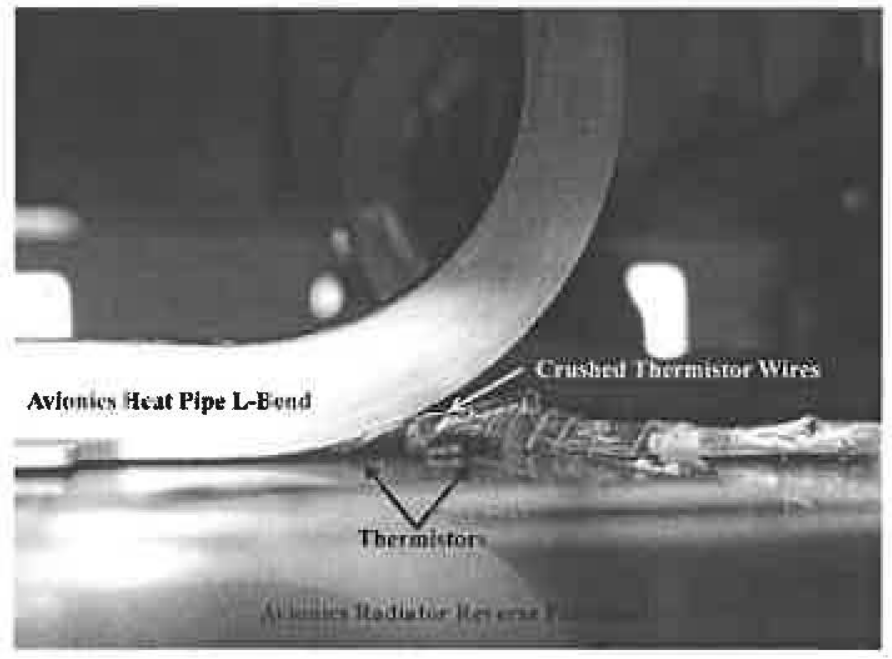

Figure 4. Avionics radiator thermistor wire interference with avionics heat pipe during integration

For accurate measurement of temperature, a thermistor must have its bead directly in contact with the surface in which it is measuring at all times, and cannot have any epoxy material separating the bead from the surface. Ideally, thermistors should be bonded on a flat surface with epoxy applied on all sides of the bead except the center, which directly senses the surface; the wet epoxy should then be allowed to dry undisturbed for at least 24 hours. However, during the installation of thermistors on the flight spacecraft, thermistors were bonded on surfaces in non-ideal orientations, many times either perpendicular or upside-down with respect to the floor. Due to schedule restrictions which required other subsystems to concurrently perform their component integrations with the thermistor installation, it was also not feasible for the spacecraft to be rotated into an ideal position for certain thermistors to be bonded. Therefore, in this installation, since Stycast 2850, Cat 9 was used for bonding, the relatively thin consistency of this epoxy caused it to run down the sides of the spacecraft, lifting the thermistor bead away from mounting surface. Therefore, there was poor contact between the bead and the surface, resulting in inaccurate temperature measurements from the thermistor. The corrective action was to remove the previously installed thermistors and re-bond with Eccobond 285, Cat 23LV. Since the Eccobond contains Cabosil to thicken the consistency of the epoxy, the new thermistors could be mounted to surfaces with non-ideal orientations without running. Lesson Learned: When bonding thermistors on inclined surfaces, use Eccobond 285, Cat 23LV or similar thick bonding agent to minimize the possibility of the epoxy running and causing poor contact between the thermistor bead and the surface.

Originally in the GPM design, Nusil CV-2946 was suggested to be used as the interface material between the avionics heat pipes and the avionics radiator. Since Nusil CV-2946 is applied as a liquid via syringes then allowed to dry after application, it conforms to the shape of the interface and therefore provides better thermal contact. However, as Nusil contains silicones and requires a wet application, this poses a contamination risk. Furthermore, the integration of the avionics radiator to the heat pipes requires that both the reverse facesheet of the radiator and the heat pipe flange interface be perpendicular to the floor. Thus, with the possibility of the Nusil running as well as the significant contamination risk, it was decided that Nusil was too difficult to be applied for this interface, and instead sheets of eGraf HITHERM 1210 were to be used instead. However, as the original design called for Nusil, the interface thermal conductivity was reduced with the use of eGraf and this caused erosion of the thermal margin 
in the design. Lesson Learned: Nusil is not ideal as a thermal interface material to integrate on surfaces at nonoptimal orientations.

The design of the U-shaped heat pipe and S-shaped heat pipe for the avionics module, and the two L-shaped heat pipes for the Power System Electronics (PSE) box, was established with little consideration of each heat pipe's ability to support its own weight when one flange was allowed to suspend freely without external support. During integration of these avionics heat pipes, all the heat pipe flanges which interfaced with the avionics module (AM) were initially bolted into their respective places on the AM structure. However, since the avionics radiator would be installed later, all the heat pipe flanges which interfaced with the avionics radiator had no support; nor could they be cantilevered off the L-shaped bends of each heat pipe either as the weight of the flanges would possibly break these L-bends. Hence, $\mathrm{C}$-shaped aluminum channels which bolted to the avionics module structure on either side of the AM cavity were required to support these heat pipe flanges when the avionics radiator was not installed. By bolting the heat pipe flanges into these channels, the load was taken off the L-bends; the channels also served as a protective cover to prevent personnel working on the spacecraft from accidentally hitting the flanges and damaging them, or causing an ammonia leak from the heat pipe. However, the necessity of these protective covers would be reduced if the heat pipe design was more structurally sound. Lesson Learned: Heat pipes should be designed to be able to support their own weight since support structure for flimsy heat pipes adds to the complexity of their integration.

Often a second thought during spacecraft design is the Multi-layer Insulation (MLI) accommodations. If thought about early enough, moving interfaces that need blankets (like gimbal motor housings) can have built in flanges that prevent the MLI from interfering in the moving interface. Also often not thought about are MLI blanket structural supports. Especially in a spacecraft like GPM, where the MLI "tents" over the electronics boxes, mechanical supports are necessary to prevent wide areas of unsupported blankets that could be unstable during the vibration of a launch. Lastly ground points are often not thought of during the design phase. A large distribution of tapped holes on the spacecraft to attach MLI grounding lugs will save time and hardship later. Lesson Learned: MLI grounding, mechanical supports and mechanical interfaces should be considered during the design phase of a project in order to make MLI integration less complex.

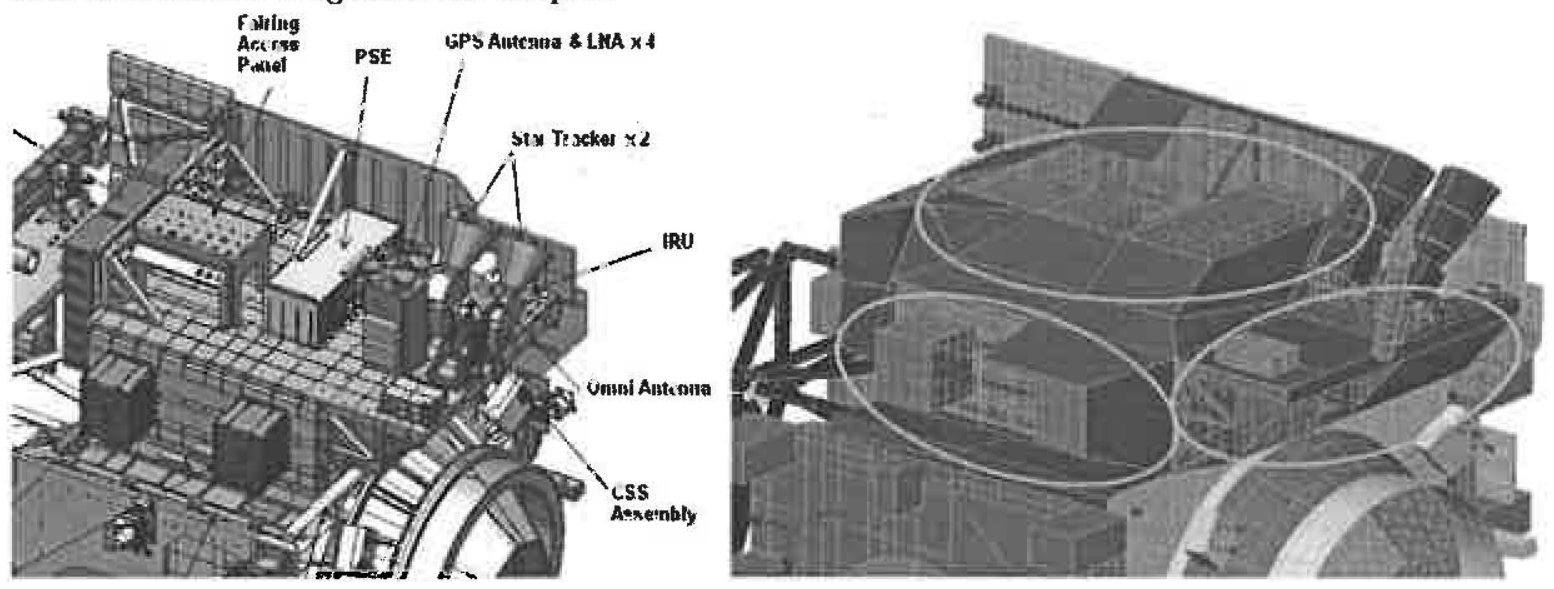

Figure 5. Left is mechanical model of avionics area, right is "tented" MLI (some MLI removed for clarity)

\section{Lessons Learned from testing the thermal flight hardware}

The GPM Observatory Thermal Vacuum (TV) test setup requires that the spacecraft be mounted to an aluminum L-Frame at the aft end. Since both the GPM Microwave Imager (GMI) and the Ku-band Precipitation Radar (KuPR) instruments occupy the front end of the spacecraft, this end could not have any ground support equipment (GSE) attached. This has resulted in multiple complications during preparation for the thermal vacuum test due to the spacecraft being cantilevered. Among these, levelness of the spacecraft is a major concern to ensure that all spacecraft heat pipes in the X-Y plane operate during test. Consequently, it was critical for the GPM project to rigorously maintain levelness throughout the duration of the test. For this purpose, the GPM project both obtained vacuum-compatible inclinometers and titanium supports for mounting between the spacecraft and test fixture. With attached three-axis inclinometers, multiple levelness measurements can be obtained during the integration of the 
GPM TV test setup, and after the thermal blanket integration the blanket configurations do not need to be broken to allow for new measurements. Furthermore, the inclinometers can be used to measure levelness inside the thermal vacuum chamber during pump-down or transitions to cold or hot temperature plateaus. In addition, 12 titanium spacers were acquired by the GPM project for both supporting the spacecraft to prevent a large sag on the front end, and for thermal isolation of the spacecraft from the test fixture. However, due to the difficulty of obtaining and machining titanium, the titanium spacers were costly to procure. Overall, though, such meticulous, constant monitoring of the spacecraft levelness would not be necessary if the spacecraft was not cantilevered, with heat pipes near the forward end at risk of not being able to start because of levelness concerns. Lesson Learned: For a large spacecraft, it is beneficial to support the spacecraft via multiple attachment points to the test fixture.

The High Gain Antenna System (HGAS) for GPM sits atop a dedicated composite deck which contains, among other components, the launch release mechanisms for the HGAS boom. For observatory-level thermal vacuum testing, it was determined to be mechanically difficult to implement any thermal test panels onto this HGAS deck. The panels could not be mounted to the deck as it cannot bear any significant loads other than those already incorporated into the spacecraft design. If the thermal panels were integrated into the test fixture design, they would need to be cantilevered over the HGAS deck to significantly influence the thermal test environment around the High Gain Antenna; this is both difficult to assemble and poses a risk to colliding with flight hardware if the support test structure fails. After iterations of the thermal vacuum test fixture design, it was determined that thermal panels around the High Gain Antenna structure would be exceedingly complex to implement and instead, only test and flight heaters already installed on the HGAS would be used to maintain temperatures within acceptable limits. Since the HGAS assembly was already qualified during system-level thermal vacuum and thermal balance testing, there was no need to test the instrument further during observatory-level thermal testing. Therefore, this allowed savings in both the complexity of the thermal vacuum fixture and the amount of test GSE, including thermal conditioning units (TCUs), required for the observatory-level thermal vacuum test. Lesson Learned: Complex subsystems may benefit from full qualification during system-level thermal testing, to reduce the need for qualification (and therefore added complexity to the test fixture) during observatory-level testing.

Scheduling conflicts with thermal vacuum chamber availability at NASA Goddard Space Flight Center necessitated that the vibration test portion of the GPM Observatory's environmental test phase be placed after the thermal vacuum testing. This posed multiple challenges and risks, as normally for any program thermal vacuum testing is the last phase of environmental testing. With vibration testing occurring afterwards, the functionality of the spacecraft and all its systems cannot be rigorously verified via thermal vacuum testing before launch, and any system that may have been damaged or broken from vibration testing may be overlooked. Furthermore, vacuumcompatible accelerometers are required to be placed onto the spacecraft before thermal vacuum testing, as the blanket configuration cannot be broken between thermal vacuum testing and vibration testing to install the accelerometers. Hence, the risk for failure increases by performing environmental testing out-of-sequence. Lesson Learned: Do not perform vibration testing after thermal vacuum testing unless absolutely necessary.

During resistance and pinout checking of the thermistor connectors, it was found on two separate instances that the thermistor wires were inserted into the wrong corresponding sockets. In the first instance, it was found through resistance testing that five pins which terminated thermistor wires were shifted one socket to the left. The error was determined be a result of a miscount by the technician completing the work because of the complex, 104-pin configuration connector used. In the second instance, it was found through touch testing that the pins corresponding to two different thermistors were accidentally switched on both the primary and redundant connectors. To correct this, the pins were swapped back to their intended sockets and a touch test was used to confirm that the pins were in their correct locations. This mistake emphasizes the importance of touch testing: if only resistance testing was used, the resistance measurement would solely have verified that there was a continuous circuit from the connector to the thermistor bead. However, it would not have verified the location of the thermistor since the resistance measurement for all thermistors are relatively equivalent. Lesson Learned: Pinout checking and resistance testing on connectors, especially when used in conjunction with the touch testing, is crucial to verify the workmanship and location of flight thermal sensors on the spacecraft.

During the testing of subsystems it was discovered that the pictures taken of the thermocouple installation were inadequate. Close up pictures of thermocouples with no surrounding context provided little insight into the location. During the High Gain Antenna Subsystem level thermal vacuum test the project photographer took pictures of the test setup including pictures of the thermocouples. Without precise instruction or active oversight, the photographer 
took the close up pictures, and then the areas became inaccessible because test blanketing was installed. The only indication of location for many of the thermocouples was the rough sketch that the thermocouples were installed by. Lesson Learned: Provide precise instruction on how to photograph the test hardware or oversee the photography in order to guarantee pictures that are meaningful to the thermal engineer.

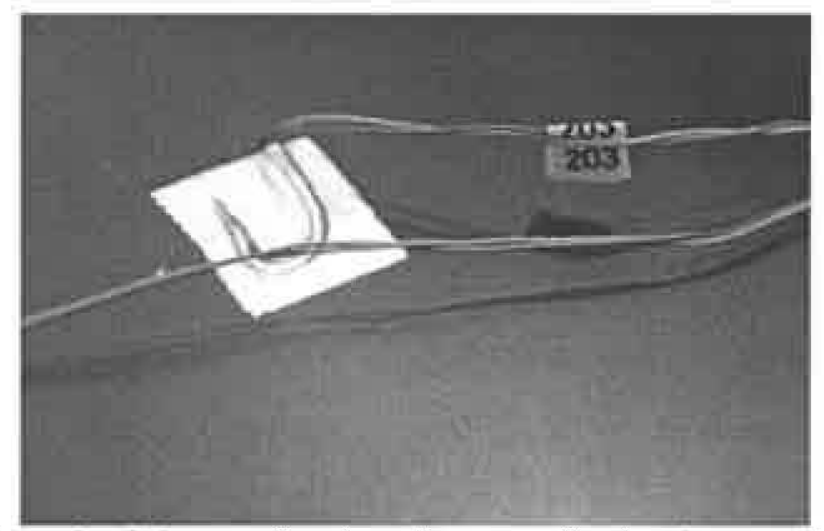

Figure 6. Example of photography of test thermocouples that does not show location.

\section{Conclusion}

The I\&T phase of GPM and the issues that arose with hardware afforded insights that can help with future satellite integrations. These insights were sorted into three major categories. 1) The specification of flight hardware using analysis results and the available mechanical resources. 2) The integration of thermal flight hardware onto the spacecraft. 3) The preparation and implementation of testing the thermal flight hardware via touch tests, resistance measurements and thermal vacuum testing. In the first category, it was found that consideration to the storage of shipping containers, using primer on aluminum parts which does not need to be removed before bonding, and reducing the number of unique parts in thermal hardware proved extremely helpful to the acquisition of flight hardware. In the second category, the "lessons learned" indicated that extra consideration should be taken on the placement and choice of thermal hardware in areas that are difficult to work with or have non-optimal orientations. In the third category, it was found that qualification during system-level testing, multiple supports for the spacecraft on the test fixture, electrical testing of the thermal hardware, and performing pre-TV vibrational testing are crucial steps to simplify the complexity of the observatory-level thermal vacuum test. It is hoped that the knowledge gained from GPM's integration and testing process will benefit and streamline future integration processes on other spacecraft programs.

\section{Appendix}

Lessons Learned are summarized here in a concise list:

1. Specify shipping containers for heat pipes that are easier to store, such as a vertical box or a box with a minimal footprint.

2. For metallic surfaces that will need to be bonded to, BR127 primer is more desirable as a passivating coating than Iridite.

3. If a conscious effort is made to use several of one type of heater or thermostat for multiple locations it simplifies the ordering processes as well as minimizes the number of flight spares that is needed.

4. When mounting thermal hardware in areas with space restrictions or "stay-out zones", care should be taken to specify the direction in which the wires route from the thermal hardware such that the risk of interference with other future hardware to be mounted is minimized.

5. When bonding thermistors on inclined surfaces, use Eccobond 285, Cat 23LV or similar thick bonding agent to minimize the possibility of the epoxy running and causing poor contact between the thermistor bead and the surface.

6. Nusil is not ideal as a thermal interface material to integrate on surfaces at non-optimal orientations. 
7. Heat pipes should be designed to be able to support their own weight since support structure for flimsy heat pipes adds to the complexity of their integration.

8. MLI grounding, mechanical supports and mechanical interfaces should be considered during the design phase of a project in order to make MLI integration less complex.

9. For a large spacecraft, it is beneficial to support the spacecraft via multiple attachment points to the test fixture.

10. Complex subsystems may benefit from full qualification during system-level thermal testing, to reduce the need for qualification (and therefore added complexity to the test fixture) during observatory-level testing.

11. Do not perform vibration testing after thermal vacuum testing unless absolutely necessary.

12. Pinout checking and resistance testing on connectors, especially when used in conjunction with the touch testing, is crucial to verify the workmanship and location of flight thermal sensors on the spacecraft.

13. Provide precise instruction on how to photograph the test hardware or oversee the photography in order to guarantee pictures that are meaningful to the thermal engineer

\section{Acknowledgments}

We would like to acknowledge the GPM I\&T and Thermal teams. Even though this paper mentions what "went wrong", that is the small portion of the thermal I\&T, the majority of tasks that went smoothly. We would like to thank GPM project management and the GPM thermal lead, Carlton Peters for supporting this paper. 